'DIE GLÖCKSELIGE EINHEITLICHKEIT DES WEIBES' UND "WOMAN IS PERFECT' - LOU ANDREAS-SALOMÉ UND H.D. IN DER SCHULE BEI FREUD.*

\title{
Gisela Ecker
}

Nachdem von seiten der Frauen nun schon seit gut 15 Jahren die Diskussion um Freud und die Psychoanalyse lebhaft und kontrovers geführt worden ist, erscheint es mir interessant, sich der frühen Rezeption durch die Zeitgenossinnen zuzuwenden. Die bürgerlichen Frauenbewegungen nahmen die Psychoanalyse kaum oder sehr obexflächlich zur Kenntnis und fallten in Rahmen ihrer pragmatisch orientierten Gleichheitsdebatte vorwiegend negative Urteile. Demgegenüber ist zu erwarten, daB sich Vertreterinnen der Avantgardebewegungen, denen ja eine besonders enge Verbindung zwischen Lebenspraxis, Wissenschaft und Kunst nachgesagt wird, intensiver mit dieser neuen Wissensmenge, die so viele Aussagen über Weiblichkeit enthält, auseinandersetzen.

Zwei Schriftstellerinnen aus ganz verschiedenen Kulturkreisen haben ihre Begegnung met Freud und der Psychoanalyse als einschneidendes Ereignis in ihrem Leben bezeichnet und dieses auch in umfangreichen Texten beschrieben: Lou Andreas-Salomé (1861-1937) und Hilda Doolittle (H.D. 1886-1961). Die in Rußland aufgewachsene deutschstänmige Lou Salome, deren Schriften auf religionsphilosophischem, literarischem und literatur-kritischem Gebiet lagen, war begehrtes Mitglied von Bohème-Zirkeln in Berlin, München und Wien um die Jahrhundertwende; die Amerikanerin H.D., Verfasserin innovatorischer lyrischer und erzählender Texte, stand am Rande verschiedener Avantgardebewegungen in den europäischen Metropolen, vor allem London und Paris. Über das turbulente Leben der beiden Frauen, das sie selbst als experimentierend verstanden, ist mehr geschrieben worden als über ihre Texte, mit einer Mischung von Reiz und Horror vor unkonventioneller Weiblichkeit, ein Phănomen, das sich im Zuge einer feministischen Kulturkritik unschwer als Fortschreibung geschlechtsspezifischer Muster erklăren läßt. Ironischerweise verdanken wir die sorefaltige Behandlung ihrer Schriften gerade der Tatsache, daB beide als Begleiterin und Geliebte bedeutender Mănner galten. Nietzsches und Rilkes im einen Fall, Pounds, Aldingtons, Lawrence im anderen, um nur die bedeutendsten unter ihnen zu nennen. Dem wachsenden Interesse an der Literatur von Frauen und der feministischen Literaturkritik, besonders in bezug auf H.D., ist es zu verdanken,

-Diesem Artiked liegt ein Vortrag zagrande, den ich 1987 auf der Tagung der Ma.A in San Francisco gehalten habe. 
daß heute ibr umfangreiches Werk zur Kenntnis genommen wird, daß H.D. langsam nicht mehr ausschließlich als das Geschöpf Pounds und als Geliebten Rilkes und 'Nin des Imagism gilt und daß Salomé außer de anderes darstellt.

Lebensläufe allerdin zeitgenössischen 'Normalbiographien', boten die Heiratsanträge an Lo Stoff für die beliebten Legenden. Abgeschmetterte Heiratsanträge an Lou von Nietzsche und andere, die nicht 'vollzogene' pikanten Dreieckenn, die von ihr abrupt abgebrochenen Affären und die phantasierte Promiskuitant die vielen Reisen Lous, die vollzogene und schen Zusammenbrüche, alles das, ihre Homosexualität und ihre psychiabenteuerlich akrobatische Geschichten eine Jongliermasse, aus der meint, aus den Legenden die 'weschichten produziert werden. Doch wer oft postuliert wird, irrt die 'wahre' Gestalt herausschälen zu können, wie der autobiographischen ebenfalls, denn welche Quelle, einschließlich , keine Fiktionen Qulthe BewuBtsein der geschichtenproduzierenden Alleine das der eigenen und der sorgfaltige Umgang Bewegungen, einschließlich Für keine der beiden Frauen bedeung damit kann weiterhelfen.

analyse den Ubertritt zu einer Schule 'te die Begegnung mit der Psychofand erst in reiferem Alter einer Schule 'mit fliegenden Fahnen', denn sie kennenlernte und Alter statt (Lou Andreas-Salomé war 50, publiziert haten. 47 Jahre alt) und nachdem beis publiart haten. Salome sagt von sich, ihr bisheriges diese Begegnung hin bewegt, und auch Lektüre bestens vorbereitet. Im Gegen H.D. war durch intensive Freudlich orientierten ersten weiblich Gegensatz zu den mehr naturwissenschaftMelanie Klein, Karsten weiblichen Psychoanalytikern wie Helene Deutsch von der geistes aren Horney fühlten sich beide Schriftstelleringentsch, richtung angezwissenschaftlichen Seite dieser januskof richtung angezogen, auch wenn Salomé die jauskopfigen neuen Denkeingelesen hatte, ein eher wissenschaftic die sich autodidaktisch intensiv Freud 1911 wăhrend eines psychschaftliches Interesse bekundete. Sic lemte 1912 Zutritt zum Mittwes psychoanalytischen Kongresses kennen, bekam Jahren entwickelte Mittwochabend-Kolleg in Freuds Wohnnen, bekam über eine Reihelte sich eine Freundschaft mit Freud und kleineren Literaturprodun abgesehen, wandte sich Salome Briefe. Von ein paa vor diesproduktion ab (fast alle der nach Salomé in der Folge von der vor dieser Zeit entstanden) und der nach 1911 gedruckten Texte waren unorthodoxe Laienanalytikerin und der Psychoanalyse zu, wurde waren psychoanalytische Schrifterin mit recht erfolgreicher $\mathrm{zu}$, wurde sogar Texte auf die Psychohriften. Insgesamt beziehen sich folgende und verfaBt (1931), der Briefwecheel und ihren Begründer: Meinende gedruckte ihrem Lebensrickblick (1951), In der Schule 1936, zwei Abschnitte in
Jahres $1912 / 13$ (1958) und sechs psychoanalytische Artikel.

Auf Arregung ihrer Lebensgefährtin Bryher und des Analytikers Hanns Sachs hin machte H.D. bei Freud eine Analyse, 80 Stunden vom 1. März bis 15. Juni 1933 und von Oktober bis Dezember 193425 Stunden. 1944, während der 'Blitz'-Angriffe in London, schrieb sie ihre Erinnerungen an diese Analyse unter dem Titel 'Writing on the Wall' auf und erganzte diese später durch 'Advent', ihre Aufzeichnungen während dieser Analyse, zusammen Tribute to Freud (1974). Ihr Gredicht über Freud, 'The Master' (1933), wollte sie zu Lebzeiten auch auf Druck hin nicht veröffentlichen: 'I will not, NOT, NOT have my analysis spoiled again' (1984: xxiv). Anspielungen auf Freud und seine Lehre durchziehen ihr gesamtes umfangreiches Spätwerk. Es gilt als gesichert, daß sie, wenn auch nicht psychisch 'geheilt', in der Analyse ihr Selbstverständnis als Dichterin zurückgewinnen konnte.

Nur auf den ersten Blick erscheinen die verschiedenen Rollen klar getrennt: hier der Analytiker, dort einmal die (gut) zahlenden Patientin, und zum anderen die berïhmte Zeitgenossin, die bei ihm Psychoanalyse studieren will, denn auch H.D. sah sich zeitweise als seine Schulerin und beschaftigte sich ausgiebig mit den Schriften der Psychoanalyse, und Salomés explizit der Wissenschaft dienendes Verhăltnis zum Meister war doch geprägt von klassischen Mustern der Übertragung. Für ihn selbst nahmen beide Frauen eine Sonderstellung ein, 'Frau Lou' als Freundin und 'Versteherin par excellence' (Freud 1966: 311), die nicht unbedingt nach wissenschaftlichen Kriterien vorzugehen brauchte; H.D. als Dichterin, deren Talent es zu bewahren und zu verstărken galt und das Vorrang vor psychischer Gesundheit hatte, ein besonders typischer Konflikt aus den frühen Jahren der Psychoanalyse.

Die Texte, die ich bentutzte, um herauszufinden, wie sich diese beiden Frauen auf die Psychoanalyse einließen, sind vielschichtig und reich an phatischen Elementen. Es ist nicht zu übersehen, daB es sich um persōnliche Beziehungen gehandelt hat, in denen vor allem von weiblicher Seite viel Intimes eingebracht wurde. Vieles in der Forschung zu beiden Autorinnen zeigt, wie verletzlich das Material ist, mit dem ich mich hier beschaftige. So kann zum Beispiel der Autor der deutschsprachigen Ausgaben von Tribute kaum seine Lust zur Reduktion des Texts auf eine psychoanalytische Fallgeschichte zugeln: 'Aber es ist beinahe zu verlockend, H.D. hinter die Schliche zu kommen; es fält schwer, statt dessen von ibr zu lernen' (Doolittle ä975: 22). Gerade das aber will ich hier versuchen. 
'Liebste H.D.' ... 'Meine liebe Lou' ... 'Lieber Professor': die phatische

'There was an argument implicit in our very bones', schreibt H.D. (1974: 13), aber sie unternimmt dennoch keine argumentative Abgrenzung. Auch für Lou Andreas-Salomé muß die Beziehungsebene 'stimmen'. Dort, wo sie Freud widerspricht, in Artikeln und Briefen, vollbringt sie wahre Mine ihrer Methen der Harmonisierung von eigentlich Unverträglichem. Eine ihrer Methoden, ohne den Anschein eines Dissens etwas Abweichendes zu sagen, ist die Umgewichtung von Freuds Worten, indem sie Bestätigung Aussagen des Meisters bevorzugt an denjenigen Stellen zur komm modalitat umschaltet. In einem Brief er auf eine andere Rezeptionsdrängenden Frafer. 'I einem brief von 1917 antwortet er auf eine ihrer Fragezeichen austeilen, immer getan habe: sie, sondern tun, was ich mit Ihren Anmerkungen 318). Salomés Abweichungen und auf mich wirken lassen' (Freud 1966: Gewand der Abweichungen von der Freudschen Lehre, die sie selbst im Kollond der Zustimmung äußert, hätten ihr, wäre sie die sie Kollege gewesen, den sicheren AusschluB Freud gerade den deutlichsten Puluß beschert. Paradoxerweise werte Bindung um:

Es ist ganz unverkennbar, wie Sie mir jedesmal voranciben Sie sich seberisch bemuhen, meine Bruchesmal voraneilen und mich ergänzen, wie Eindruck, diese sei so in besonderem AusmaBe, seitdem ich ergänzen. Ich habe den ibido in Gebrauch gezogen habe. Ohne diesentem ich den Begriff der narziBt. enteilt zu den Systembauern, zu Jung ode diesen, meine ich, wären auch Sie mir Hinter den eingehaltenen kulturspezer eher zu Adler (Freud 1966: 68),

begegnung mit ihren kavaliershaften losung, die Rivalisieren auschhaften Gesten verbirgt sich eine Verharmsie die Spezifik ihrer Ausschließt. Salomé wirkt selbst dabei mit, wenn begründet sieht. So Auslegungen abmildernd als in ihrer Weiblichkeit analyse:

Wie es gewesen wäre als bloß zachliches Wissen ohne dies menschliche Eriebnis,
kann ich mir überhaupt nicht Indem sie darüberbinans vorstellen. (Bin ja auch eine Frau.) (Freud 1966: 214). Psyche zu ihrem Thema machte, befand Freud bereits als ratselhaft und undund sie sich auf einem Territorium, das und kam ihm auch daduft und undurchdringlich ins Abseits gestellt hatte Mebrfach betont Freudch nicht 20 nahe.

seinen Seminaren ist. Als sie angenehm ihm 'Frau Lous' Anwesenheit in
10.11.1912 von seiner 'Unart, den Vortrag immer an eine bestimmte Person im Hörerkreis zu richten ... und starrte gestern wie gebannt in die Sitzlücke, die man für Sie gelassen hat' (Freud 1966: 12). Als er sich viele Jahre später für eine Blumensendung bei H.D. bedankt, differenziert er zwischen Lob (das er nicht akzeptieren könnte und das eher mănnlich konnotiert ist) und Zuneigung: 'What you gave me, was not praise, was affection and I need not be ashamed of my satisfaction.' Und der alte Freud endet diesen Brief mit einer überraschenden Wendung: 'Life at my age is not easy, but spring is beautiful and so is love' (H.D. 1974: 194).

'My intuition challenges the Professor, though not in words' (1974: 99), schreibt H.D., und 'the Professor was not always right. That is, yes, he was always right in his judgments, but my form of rightness, my intuition, sometimes functioned by the split-second ... the quicker' (1974: 98). Diese Intuition gab ihr auch das Recht, ihm ziemlich untypische Aussagen zu unterstellen:

It is difficult to imagine the Professor saying solemnly: 'I stumbled on a well of living water, the river of life. It ran muddy or bright. It was blocked by fallen logs, some petrified - and an accumulation of decaying leaves and branches. I saw the course least a rer and how ran, and l, persold ly, clear (1974: 83)

So poetisch transformiert ist er sonst nicht behandelt worden, und auch die mythologischen Personae Theseus oder Asklepios, die er in ihrer Phantasie verkörpert, sind in ihrer Dichtung mehr als Figuren der Übertragung im analytischen ProzeB. Der phatischen Ebene wird in Tribute viel Aufmerksamkeit geschenkt, den 'Schwatzstunden', dem gemeinsamen Betrachten von Freuds 'goods/gods', d.h. seiner kleinen antiken Statuen. Die Harmonisierung, die als Produkt historisch spezifischer weiblicher Sozialisation die Schriften beider Frauen prägt, kann trotzdem über die eigenwilligen Wege, die beide in der Bewertung psychoanalytischer Ansătze gehen, nicht hinwegtäuschen; der Widerstand kommt in gefalliger Kleidung einher.

\section{'Die glïickselige Einheitlichteit'}

Der Narzißmus ist das zentrale Thema in Lou Andreas-Salomés psychoanalytischen Schriften und gleichzeitig der Angelpunkt ihrer Abweichung von Freud. Die Diskussion darüber beginnt bereits 1913 in ihrem Tagebuch, ist eines der wichtigsten Themen in ihrem Briefwechsel mit Freud und findet schließlich 1921 mit Salomes Artikel 'NarziBmus als Doppelrichtung' in der Zeitschrift Imago ihren wichtigsten Ausdruck. Freud hatte in seinen Vorlesungen und in einem Aufsatz von 1914 vom reifen Individuum die Verlagerung der Libido von der Selbstliebe zur 
Objektliebe verlangt und dieser Objektbesetzung nur einen eher unvermeidlichen Rest von ursprünglichem Narzißmus zugestanden. Im Gegensatz dazu setzt sich Lou Andreas-Salomé in immer neuen Ansätzen und mit leidenschaftlicher Emphase für die positive Bewertung alter Formen von NarziBmus im erwachsenen Ich ein, vor allem aber für der Formen schen Anteile an der Objektiebe und die narziß aber für die narzißtiweiblichen Geschlechts.

Zweierlei ist ihr an dieser narzißtischen Libido wichtig, nämlich daß sie eine Rückbeziehung darstellt und daß sie eine Form von Verschmelzung ist. Die erste Phase der Selbstliebe des primä Form von Verschmelzung der ungespale Bezies chenden Allmach symbiotischen Verschmelzungszustand mit entspre"liebt" " (1917: 155) Defuhlen. Das Kind "lebt die Mutter, ehe es die Mutter liebt (1917: 155). Dieser prä-ödipale Idealzustand - darüber sind sich so unterschiedliche Theoretiker/innen wie Freud, Kristeva, Lacan, Winnicott und Kohut einig - wird mit dem Eintritt in das Symbolische und die Sprache unwiderruflich aufgegeben. Der Unerfüllbarkeit des entspreräuen Begehrens ist sich Salomé zwar grundsätzlich bewußt, und sie raumt ein, daß die 'Wiederverschmelzung', wie sie es nennt, durch Sublimation Kompromisse eingehen muß, doch stellt sie nennt, durch nach eine unersetzliche positive Kraftquelle stell sie ihrer Meinung lebnis der Allteilhaftigkeit' (1921. 374) zur Verfügung, das 'UrerTexte, die im schillernden (1921: 374). Die sprachliche Fassung ihrer 'Urtraum allesumfassenden Gebrauch von Komposita-Kreationen den evozieren, spicgelt diesesden, allesuntergründenden Seins' (1921: 376) problematischer Seite. Für ihre Zwecke deutet sie auch den Narziß-
Mythos um:

Ein wenig hat es der Taufpate des Terminus, der Spiegelheld NarziB auf dem Gewissen, wenn dabei zu einseitig die ichbeglückte Erotik allein haraus, auf dem sondern vor dem der Ner Narkißos der Sage nicht vor künstlichen Spiegel Abeht sich als al les noch, velleicht hätte er sonst nicht davor verweilt, sond, sondern

Bereits in ihren Tagebuchnotizen von 1913 macht sie sich Gedanken
über die schöpferischen Mir

Mebensunreife, sondern auch der Narzibmus ... nicht nur eine zu überwindende bloß die Grenze, tiber die meine wesenserneuernde Lebensbegleitung ist, also nicht auch die, wo das Ineinander analysierend nicht mehr hinäberkommt, sondern überpersóntich und deanalber von Ich und Libido schopferisch, d.b. insofern zergfiederbar und logisierbar ist (1983: 185)
Freud sieht hinter solchen eigentlich ketzerischen Ideen nicht ohne implizite Kritik ihre

Kunst, über das Gesagte hinauszugehen, es zu vollenden und bis zu einem fernen Treffpunkt konvergieren zu machen ... Natürlich gehe ich nicht gleich mit ... Was mich interessiert, ist die Scheidung und Gliederung dessen, was sonst in einem mich interessiert, ist die Scheidung und Gliederung
Urbrei zusammenfließen wörde (Freud 1966: 38).

AufschluBreich für unseren Zusammenhang sind die Verbindungen zwischen Weiblichkeit und Narzißmus, die sie zieht. Die Begründung dafür sucht sie einerseits im Anatomischen, denn der 'beharrliche Überrest der Klitorissexualität' verhindere mit der resultierenden 'infantile(n) Erogenität des Gesamtleibes' (1921: 370) die auf einen Punkt konzentrierte männliche Lust, mit der Gebärfähigkeit gewinne sie aber auch ein 'Stück Aktivităt' und habe somit Anteil an allem, sei 'darin fast doppelgeschlechtlich ergänzt, und eben drum wieder ins Urnarzißtische zurackgerundet' (1921: 370). Vieles ließe sich, in andere Worte abersetzt, in den heutigen Diskurs über weibliche Sexualität einordnen. Die Vorstellung vom narziBtischen Weiblichen hatte sie bereits ohne psychoanalytische Begründung und ohne sich des Begriffs noch bedienen zu können in ihrem Artikel 'Die in sich ruhende Frau' aus dem Jahr 1899 vertreten. Dort ist von der 'glückseligen Einheitlichkeit' des Weibes die Rede; es zeichne sich durch 'intaktere Harmonie', durch 'weibliche Selbstherrlichkeit' aus, es sei 'der genieBendere Mensch', aber auch gleichzeitig der widerspruchsvollere und weniger weit entwickelte. 'Dies Ungerechtere am Weibe, das, im traditionellen Sinn, weit Gewissenlosere, macht sie auch viel weniger kultivirbar als ihn' (1921: 303).

In seinem Narzißmus-Aufsatz gesteht Freud nur dem Mann volle Objektliebe zu, die Frau dagegen sei aufgrund der 'Steigerung des ursprunglichen Narzißmus' in ihrer 'ordentlichen' (!) Objektliebe eingeschränkt, denn sie liebe nur sich selbst. Interessant sind die Vergleiche, die er zieht, um seinen Lesern das Phänomen nahezubringen:

Solche Frauen üben den groBten Reiz auf die Milnper aus, nicht nur aus assthetischen Gründen ... Es erscheint nämlich deutlich erkennbar, daB des NarziBmus einer Person eine grobe Anziehung auf diejenigen anderen entfaltet, welche sich des vollen AusmaBes ihres eigenen NarziBmus begeben haben und sich in der Werbung um dic Objektliebe befinden; der Reiz des Kindes beruht xum guten Teil auf dessen NarziBmus, seiner Selbstgenugsamkeit und Unragănglichkeit, ebenso der Reiz gewisser Tiere, die sich um uns nicht zu Kümmern scheinen, wie die Katzen und großen Raubtiere, ja selbst der grobe Verbrecher und der Humorist zwingen in der poetischen Darstellung unser Interesse durch die narziBtische Konsequenz, mit
welcher sie alles ihr Ich Verkleinernde von ihm fernzuhalten wissen (1914: 55).

Der leibhaftigen Lou wird nachgesagt, sie habe dieser Freudschen Beschreibung Modell gestanden (cf. Kofman; Salber). Dieser wertet im 
18

selben Artikel die vielzitierten mānnlichen 'Klagen über die Rätsel im Wesen desselben' (1914: 56) als Folge dieser 'Selbstgenügsamkeit des Weibes'. Wenn die Raubkatze nun selbst zu sprechen anfängt, erscheint es, als insistiere die Frau als Subjekt auf denjenigen Wunschphantasien, die hr sonst als Objekt zugeschrieben wurden. Aler shr eigenwillige Weise, in imen wurden. Allerdings tut sie dies auf eine einer radikalen Umkentiven Ansătzen und von aller Weiblichkeit cesagt hat, grenzt sie in den späteren Auseinandersetzungen stärker auf das Mütterliche ein: 'im Mütterlichen kreuzt sich nur ollends das Ewig-autoerotische mit dem Allesuerten kreuzt sich nur Umkreis, den die Libido beschreiben, der breiteste Oxganismus' (1917: 182-3).

Indem das Weiblicbe bei ihr keinen Mangel kennt (symbolisch ausgedrückt im Penis-Neid), entfernt sich ruhenden -Neid), entfernt sich Lou Andreas-Salomés Idealbild der in des Weiblichn narzißtischen Frau entschieden vom Freudschen Konzept des Weiblichen, aber indem die Frau nicht aktiv in die Kultur entritt, folgt sie seinen Vorstellungen von der weiblichen Unfähigkeit zur Kultur. Salomé gestaltet ein Bild eigenstāndiger Weiblichkeit, das sich nicht am erscheint erstaunlich orientiert. Die Rückwendung zum Prä-Ödipalen analytischer Theorien der analytischer Theorien der Narzißmus aufgewertet wird (cf. Kohut, Stein), zum anderen weil sich heute viele Diskussionen über weibliche Sexualentscheidende Fiktions Unterscheid ist allerdings, daB Lou Andreas-Salomé den matische Notwendigkens nach Einheit verkennt und die und die traueinsehen will. Daza Julia Kristepaltung in späteren Lebensphasen nicht

We live in a civilization wheret femininity is absorbed by motherhood. If, however, one looks at it moresentation of territory; what is fantasy that is nurtured by the adult man or more losely, this

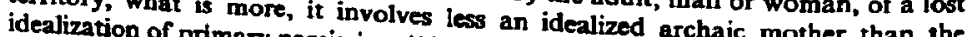
Unbewu 1 ist

muß sie doch unermüé doch dem symbolischen Einbruch Tribut zahlen, das Gemeinte suchen und nach dem rechten sprachlichen Ausdruck für begreiflicherweise nicht im Lexikon dann oft nur mit Wörtern tun, die

\section{'A woman's langhter'}

Für Lou Andreas-Salome

schlußstelle des objektiv Gültigalt 'das anscheinend Subjektivste als AnSchreiben nach diesem Prinaip.en' (1921: 382); H.D. verfuhr in ihrem
19

erkennbar an autobiographischem Material orientiert (überdies ermutigte Freud sie dazu, weiter von diesem auszugehen), und doch geht es in diesen prozeßhaften Texten, die durch und durch konstruierte Kunstprodukte sind, nicht um Repräsentation von 'Leben', das Erzählen oder Aufarbeiten bereits vergangener Ereignisse. H.D. holte sich bei Freud Bestätigung für ihre Sicht des Subjekts als Schnittstelle des Allgemeinen, für ihr visionăres Vorgehen und ihren Weg hin zu einer esoterischen Spiritualităt. Dies konnte nur durch eine Umbewertung seiner Aussagen geschehen, denn während es Freud darauf ankam, Unbewußtes bewußt zu machen, zielte H.D. umgekehrt darauf, die Zugehörigkeit alles dessen, was ans Bewußtsein getreten war, zu einem einzigen kollektiven UnbewuBten aufscheinen zu lassen, das eine gewaltige synthetische $\mathrm{Kraft}$ besaß und auf keinerlei Gesetze der Materie, des Raums und der Zeit Rücksicht nimmt. Für sie stellt selbst Freud mit seiner analytischen Vorgehensweise nur einen kleinen Teil dieses großen Zusammenhangs dar. H.D.s Freud ist einer, dem man sonst nicht begegnet:

He had dared to say that the dream came from an unexplored depth in man's consciousness and that this unexplored depth ran like a great strcam or ocean underground ... He had dared to say that it was the same ocean of universal consciousness, and even if not stated in so many words, he had dared to imply that this consciousness proclaimed all men one; all nations and races met in the universal world of the dream (H.D. 1956: 71).

Eine Sehnsucht nach ungeteilter kosmischer Ganzheit durchzieht bereits diese Eindrücke, die in einer utopischen Vision über das Persönliche hinausgehen: der Mensch wirde in diesem umfassenden Traum 'forgo barriers of time and space, and man, understanding man, would save mankind' (1956: 71). Auch in ihrer Fiktion ist sie die Dichterin der verwischten Grenzen zwischen Figuren, der narzißtischen borderline-Fälle (cf. Ecker 1987), und auch ihre bereits ausgebildete literarische Technik kommt den auf der Couch geltenden Prinzipien der freien Assoziation und der Verbindung von oberflächlichen Dissoziierten weit entgegen.

Künstlertum und Weiblichkeit waren für Lou Andreas-Salomé unverträglich, obwohl sie zwischen dem männlichen Künstler und der Frau schlechthin eine innere Verwandtschaft erkannte denn wie der Künstler lebe die Frau aus der Fülle. Allein der Narzißmus des Künstiers ermögliche es ihm, 'aus tiefer Identifikation mit allem herauszuschaffen' (1921: 384), und trotz aller damit verbundenen Gefahren sieht sie in der Kunstproduktion 'ein Schweigen aus dem Vollen, worin Rausch und Frieden sich zur gleichen unerhörten Erfahrung einen' (1921: 386). Ähnlich narziBtisch ausgerichtet, aber weniger ausdifferenziert, sei das Kunstlerische im Weibliche eher rückbezüglich, 'es ist als kreise in ihm das Leben gleichsam in seiner eigenen Rundung' (1899: 296). Konsequenterweise 
betrachtete sie ihr eigenes literarisches Schaffen eher abwertend als private Betätigung, die nur aus Grïnden des Broterwerbs zu einer Veröffentlichung führte. Für H.D. dagegen war weibliches Künstlertum ein zentrales Anliegen und Gegenstand ihrer Analyse bei Freudum ein Gedicht 'The Master', das erst 1981 veröffenticht warde (Friedrem DuPlessis 1981 und H.D. 1984:451-61), dafür.

"And it was he himself, he who set me free/to prophesy ... no,/he was rather casual, $/$ "We won't argue about that" (he said) $/$... no, he was Wir dürfen allerdings nicht vergessen handelt, daß diese Worte von der Persom Lyrik Referenzbezug fuir den 'Man der Persona eines Gedichts stammen. Als es nicht angemess den 'Master' ist zwar Freud identifizierbar, jedoch wăre Geschit angemessen, den Text nur als Besprechung eines abgeschlossenen Geschehens zu benützen. Vielmehr wurde die Dich erin abgeschlossenen lyrisches Szenario zu erschaffen, in dem die Sprecherin dazu angeregt, ein Meister folgt und ihm Widerstand leistet Sprecherin abwechselnd dem weiblichen Idealfigur als Reaktion leistet und in dem sie das Bild einer nisch evoziert. Die Personeaktion auf die Freudsche Weiblichkeit hymvon Geziert. Die Persona des Gedichts entwickelt ein breites Spektrum von Gefühlen, von Dankbarkeit und idealisierender Verehrung für den der im Besitz von 'measureless Truth' ist, bis zu Zern was angry at the old man,/ I wanted an answer,/ a neat answer', 'I was angry with the old man/ with his talk of the man-strength'. Das sprechende vom va in seiner Sexualität gespaiten ist ('I had two loves separate') erhält oine valterlichen Meister eine Erklärung für diese Spaltung und kann i vereint:
einem ersten Bild über Weiblichkeit sprechen, die Gegensătze in sich

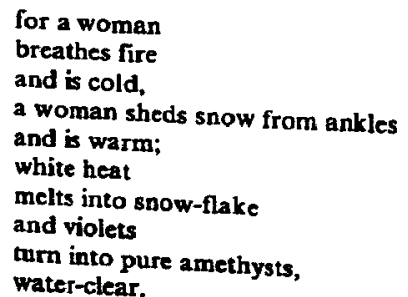

Woher die Botschaft kom

annimmt, bleibt ambig, aber' die sie dann empfangt und widerstrebend accept from wisdom/, aber 'Papa' ist daran nicht unbeteiligt: 'I could not dazu einen Verweis in Tribut love taught, / woman is perfect'. Wir finden mit Fread eine seiner Statue, und zwar eine Szene, in der $H$. favorite, he said ...' 'she is perfect' Abbild der Nike, betrachtet: ' $T$ This is my '... 'only she has lost her spear' '(1974: 69).
In Tribute versteht sie Freuds Ausspruch 'she is perfect' als Bemerkung über die klassische Perfektion der Abbildung, aber gleichzeitig wird unübersehbar die Auffassung vom weiblichen Mangel mit angesprochen ('she has lost her spear'). Das Weibliche ist doppelt eingebunden, in die perfekte (männliche) Repräsentation und Bilderproduktion und in die normative Auffassung vom weiblichen Geschlecht. Dies wird ironisch im Subtext aufgedeckt. Wenn nun die Autorin in 'The Master' postuliert, 'woman is perfect' und dies auch noch im Bild der ekstatischen Tänzerin ausführt, so erhält dieses Bild den Charakter eines teilweise noch vom Master autorisierten Gegenentwurfs,

$$
\begin{aligned}
& \text { for she needs no man, } \\
& \text { herself } \\
& \text { is that dart and pulse of the male, } \\
& \text { hands, feet, thighs, } \\
& \text { herself perfect }
\end{aligned}
$$

der die Bedingungen der Folie, von der er sich ablösen will, noch in sich trägt. In narziBtischer Selbstbezüglichkeit und nicht ohne Allmachtsphantasien ('she is a woman, / yet beyond woman, / yet in woman') verein die imaginierte weibliche Figur männliche und weibliche Züge in sich, und aus der Fülle entsteht 'a woman's laughter'.

Claire Buck (1983) kritisiert zu Recht die Auffassung von Friedman und DuPlessis und mit ihnen diejenige vieler weiterer Interpretinnen, H.D. habe hier eine genuin weibliche Sprache geschaffen und das Gedicht sei Ausdruck dafür, daß sie durch Freud zu einer ganzheitlichen weiblichen Identităt gefunden habe. Es gibt viele Anhaltspunkte im Text, die nahelegen, daB die Dichterin die Utopie der perfekten Frau nicht ungebrochen als Ziel aufruft. Die als Beweis für eine volle weibliche Subjektivitat verstandenen Passagen des Texts sind vom sprechenden Ich Subjektivitat verstandenen Passagen des Texts sind vom sprechenden Ich
distanziert, durch den Ton vom ubrigen Sprechduktus abgehoben, und auch die verwendeten Bilder ('her feet are the delicate pulse of the narcissus bud,/ pushing from earth/... reaching out/ that first evening/ alone in a forest') kennzeichnen das Gesagte als Wunschphantasien, nicht als erreichten Zustand. Das im Gedicht geăußerte weibliche richtet sich gegen die Spaltung und beschwört eine imaginäre Einheit herauf. Entsprechende Phantasien sind in H.D.s Werk stark ausgeprägt und bestimmen auch die Suche ihrer weiblichen mythologischen Figuren. Was ich dabei besonders wichtig finde, ist der Umstand, daB die Autorin nie bei diesen Bildern der Verschmelzung von Ich und Weit und des ganzheitlichen weiblictien Subjekts stehenbleibt, sondern sie als extreme Wünsche, als instabil, als Teil eines gespaltenen Selbst und nicht als utopischen Endzustand gestaltet. Die Trennung zwischen dem Imaginarren und dem Symbolischen ist als Bruch in viele ihrer Texte eingeschrieben, vor allem in den 
komplexen Beziehungen zwischen Körper und Sprache, die sie beschreibt. Sprache geschehen mu $B$, will merung, die beim Eintritt des Subjekts in problematisierten Yerschell mitberïcksichtigt werden, $15 B t$ jeden unerfüllbares Begen Verschmelzungswunsch als Regression in ein real nicht einer solchen 'erfüllten Weinen. Eine Literaturkritik, die Wunsch-Bilder weiblichen Schreibens wertet, wicheit' im Text als Ausdruck gelungenen eigenen Praxis fortschreiben, würde den NarziBmus auf der Ebene der

\section{Die narzibtische Verfahrung}

Obwohl im Zusammenhang mit Weiblichkeit eine Reihe andere Themen - so beispielsweise die Hysterie - im Umkreis der Freudschen Lehre diskutiert wurden, griffen die beiden wichtigsten (nicht primär Weise mus auf, die eine in, Uberraschenderweise übereinstimmend den NarziBin dichterischer Form essayistisch-theoretischen Schriften, die andere blicks. Der Theorie des und innerhalb eines assoziativ gestalteten Rückhabe, ein Bild von Wes Mangels setzen sie, wie ich darzustellen versucht ungespalteten prä-ödipalen Zut entgegen, das in Rückwendung auf den Vor allem im Fall von Zustand eine imaginäre Einheit beschwört. Konstrukte täuschend nahe andreas-Salomé rücken die entworfenen bilder der femme fatole une an die zeitgenössischen Zerr- und Wunschbilder der femme fatale und der männermordenden Kindfrau heran. Sie sind also nicht frei von dem in der Kultur bereiden Kindfrau heran. Sie Beim Perspektivenwechsel, diesmaltur bereits vorfindbaren Material. Stereotyp verkörpernden $F$, diesmal von innen, aus der Sicht der das Art das Begehren ist, das diese Figun allerdings erst deutlich, welcher Begehren ihr in Form von Projektion in sich trăgt und nicht, welches gebracht wird (wie et on Projektionen von männlicher Seite entgegenLulu, der Dramenfigur des Zeitgenoss aus Faszination and Abwehr, die psychischen Gewinns, den sich ditgessen Wedekind begegnet). Die Art des formuliert werden. Doch sich die Frau verspricht, mußte eben erst noch auf seine Frage 'Was will das Freud war klar gewesen, daß die Antwort Offenheit der avantgardistischen Moderne 'Phallus' lauten wirde. Die schen machte es möglich, diese erst einmal gegenüber unbewußten WünSalomé und H.D. wagten sich darin einmal zu artikulieren. Lou Andreasgeschaffenen Voraussetzingen darin weit vor. Sie konnten die von Freud nützen und setzten sich dannau für eine Exforschung dieser Wünsche tive mit seinen Ideen dann aus der vernachlassigten weiblichen PerspelWiderspritche auftauchten, ist nicht DaB dabei in erster Linie Fragen und
23

weibliches narziBtisches Streben zutage, das uns heute noch erhebliche Probleme bereitet.

'In a world felt to be especially alienating for women, this idea of psychic oneness or primary narcissism has its own peculiar force', schreibt Jacqueline Rose in einer Analyse der gegenwärtigen feministischen Positionen (1986: 102). In der Tat sind die feministischen Utopien eines ungespaltenen Selbst und der Räckkehr zur Einheit mit einer starken Mutter besonders häufig, kennzeichnen den feministischen mainstream diesseits wie jenseits des Atlantiks (cf. Gallop 1987). Da angesichts der gesellschaftlich politischen Realitäten der Eintritt in die symbolische Ordnung für Frauen so viel weniger anzubieten hat als für das mānnliche Geschlecht, liegt das Begehren nach voller, ganzheitlicher Subjektivität nahe. H.D. wird im Znge dieser narzißtischen Orientierung, obwohl sie sich für eine solche Projektion gar nicht eignet, zu einer der 'key ancestresses', wie etwa bei Alicia Ostriker, die die kollektiven (in einem ungeteilten 'we' formulierten) Wüsche anmeldet: 'We want strong mothers',

... the promise of alternative vision: acts of imagination whereby we might conceive, as it were, the valleys being exalted, the crooked made straight, and the rough places plain (1986: 479).

Die Mutter dieser idealisierten Dyade soll den Weg ebnen, alle Hindernisse wegräumen und vor allem die Töchter beruhigen, daß der Umweg über die Trauer der Trennung (und der unzureichenden Angebote für die Leistung der weiblichen Trennung in unseren - westlichen - Gesellschaftssystemen) nicht nötig sei. Doch es gibt kein Ausweichen vor der unbequemen Einsicht in die unvermeidlichen Probleme weiblicher Subjektivität. Die frühen Auseinandersetzungen von schreibenden Frauen mit der Lehre Freuds fuhren einige dieser Probleme im historischen ProzeB vor.

\section{Bibliographic}

\section{Andreas-Salome, Lou}

1899 'Die in sich ruhende Frau', in: Zur Psychologie der Frau, ed. Gisela BrinkerGabler, Fischer Frankfurt, 1978: 285-311.

1914 'Zum Typus Weib', in: Imago 3: 1-14.

1917 'Psychosexualităt', in: Die Erotik. Vier Aufsätze, Ullstein, Frankfurt/Berlin 1986: 149-83.

1921 'NarziBmus als Doppelrichtung', in: Imago, 7: 361-86.

1931 Mein Dank an Freud, Internationaler Psychoanalytischer Verlag. Wien. 
1983 In der Schule bei Freud Tagebuch eines Jahres (1912/1913), Ullstein, Frankfurt/Berlin.

Binion, Rudolph

1968 'Frau Lou' - Nietzsche's Disclple, Princeton UP, Princeton.

Buck, Claire

1983 'Frend and H.D. - Bisexuality and a Feminine Discourse', in: $m / f 8$ : 53-66.

Doolittle, Hilda

1974 Tribute to Freud, Boston 1974, Manchester Carcanet 1985.

1975 Huldiging an Freud, Ulistein, Frankfurt/Berlin/Wien.

1984 Collected Poems 1912-1944, Manchester Carcanet.

DuPlessis, Rachel Blau

1986 H.D.: The Career of that Struggle, Brighton Harvester.

Freud, Sigmund

1914 'Zur Einfuhrung des NarziBmus', in: Studienausgabe Band III, Fischer, Frankfurt 1975: 37-68.

1966 Lou Andreas-Salome, Briefwechsel, Fischer, Frankfurt.

Friedman, Susan $\mathrm{S}$.

1981 Psyche Reborn. The Emergence of H.D., Indiana UP, Bloomington.

1981 DuPlessis, Rachel Blaw. 'Woman is Perfect': H.D.'s Debate with Freud', in: Feminist Studies 7: 417-30.

Gallop, Jane

1987 'Reading the Mother Tongue: Psychoanalytic Feminist Criticism', in: Critical Inquiry 13: 314-29.

Guest, Barbara

1984 Herself Defined. The Poet H.D. and Her World, Doubleday Garden City, NY.

Kofman, Sarah

1985 The Enigma of Woman. Woman in Freud's Writings, Cornell UP Ithaca, London.

Kohut, Heinz

1973 Narzißmus, Suhrkamp, Frankfurt.

Kristeva, Julia

1983 'Stabat Mater', in: The Kristeva Reader, ed. Toril Moi, Blackwell, London: Stabat Mat

Martin, Biddy

1986 ' 'Woman and Modernity': The (Life)Styles of Lou Andreas-Salome', in: University of Wisconsin-Mihwoukee Center for Twentieth Century Studies, Working paper 5.

Ostriker, Alicin

1986 'What Do Women (Poets) Want? H.D. and Marianne Moore as Poetic Ancestresses', in: Contemporary Literature 27: 475-492.

Rose, Jecquetine

1986 Sexwality in the Field of Vision, Verso, London.
Salber, Linde

Wuic unserer Kultur. Lou Andreas-Salome', in: Zwischenschritte 2: 17-27.

Stein Angelika und Herbert

1987 Kreation

1987 Kreativitat. Psychoanalytisch

Welsch, Ursula, Wiesner, Michacla 1988 Lou Andreas-Salomé. Vom 'Lebensurgrund' 